PROCEEDINGS OF THE

AMERICAN MATHEMATICAL SOCIETY

Volume 135, Number 8, August 2007, Pages 2519-2526

S 0002-9939(07)08745-X

Article electronically published on March 2, 2007

\title{
INNER SEQUENCE BASED INVARIANT SUBSPACES IN $H^{2}\left(D^{2}\right)$
}

\author{
MICHIO SETO AND RONGWEI YANG
}

(Communicated by Joseph A. Ball)

\begin{abstract}
A closed subspace $H^{2}\left(D^{2}\right)$ is said to be invariant if it is invariant under the Toeplitz operators $T_{z}$ and $T_{w}$. Invariant subspaces of $H^{2}\left(D^{2}\right)$ are well-known to be very complicated. So discovering some good examples of invariant subspaces will be beneficial to the general study. This paper studies a type of invariant subspace constructed through a sequence of inner functions. It will be shown that this type of invariant subspace has direct connections with the Jordan operator. Related calculations also give rise to a simple upper bound for $\sum_{j} 1-\left|\lambda_{j}\right|$, where $\left\{\lambda_{j}\right\}$ are zeros of a Blaschke product.
\end{abstract}

\section{INTRODUCTION}

In $H^{2}\left(D^{2}\right)$ with coordinates $z$ and $w$, multiplications by $z$ and $w$ (denoted by $T_{z}$ and $T_{w}$, respectively) are shift operators with infinite multiplicity. A subspace $M$ is said to be $z$ (or $w)$ - invariant if $M$ is invariant under $T_{z}$ (or $T_{w}$, respectively), and $M$ is said to be invariant if it is invariant under both $T_{z}$ and $T_{w}$. It is wellknown that in general invariant subspaces of $H^{2}\left(D^{2}\right)$ can be very complex (cf. $[\mathrm{Ru}]$ ), and their study demands new ideas and techniques (cf. [DP], DM], Ya1]), as well as good understanding of some examples. This paper studies a type of invariant subpace that is constructed through an inner sequence. A sequence of inner functions $\left\{q_{j}(z): 0 \leq j \leq m\right\}$, where $m$ may be infinite, is called an inner sequence if $q_{j+1} \mid q_{j}$ for each $j$. We will see that this type of invariant subspace has a simple structure, and it has direct connections with the Jordan operator.

The classical Hardy space $H^{2}(D)$ in the variable $z$ and that in the variable $w$ are different subspaces in $H^{2}\left(D^{2}\right)$, and we denote them by $H^{2}(z)$ and $H^{2}(w)$, respectively. For every $g \in H^{2}(w)$, we define an operator $\pi_{g}: H^{2}\left(D^{2}\right) \longrightarrow H^{2}(z)$ by

$$
\pi_{g}(f)(z)=\int_{T} f(z, w) \bar{g}(w) d m(w), \quad f \in H^{2}\left(D^{2}\right),
$$

where $T$ is the unit circle and $d m(w)$ is the normalized Lebesgue measure on $T$. It is easy to check that $\pi_{g}$ is bounded. In fact, one verifies that

$$
\pi_{g}^{*} h=g(w) h(z), \quad h \in H^{2}(z),
$$

Received by the editors November 4, 2005 and, in revised form, April 6, 2006.

2000 Mathematics Subject Classification. Primary 47A13; Secondary 46E20.

Key words and phrases. Core operator, Hardy space over the bidisk, Jordan operator, Blaschke product.

(C)2007 American Mathematical Society Reverts to public domain 28 years from publication 
and hence $\left\|\pi_{g}\right\|=\|g\|$. We now look at a few facts regarding $\pi_{g}$. If $M$ is $z$-invariant, then for every $g \in H^{2}(w)$

$$
z \pi_{g}(M)=\pi_{g}(z M) \subset \pi_{g}(M) .
$$

If $M$ is $w$-invariant, then

$$
\pi_{w^{i}}(M)=\pi_{w^{i+1}}(w M) \subset \pi_{w^{i+1}}(M)
$$

for every integer $i \geq 0$. So in the case when $M$ is invariant, the closures $\overline{\pi_{w^{i}}(M)}, i=$ $0,1,2, \ldots$, is an increasing sequence of invariant subspaces of $H^{2}(z)$. By Beurling's theorem, this gives rise to a sequence of inner functions $\left\{q_{i}(z): i \geq 0\right\}$ with $q_{j+1} \mid q_{j}$, i.e., an inner sequence, such that $\overline{\pi_{w^{i}}(M)}=q_{i} H^{2}(z)$. It then follows easily that

$$
M \subset \bigoplus_{j=0}^{\infty} q_{i} H^{2}(z) w^{j} .
$$

One observes that $\bigoplus_{j=0}^{\infty} q_{i} H^{2}(z) w^{j}$ is clearly $z$-invariant. Moreover, since $q_{j+1} \mid q_{j}$,

$$
w q_{j} H^{2}(z) w^{j}=q_{j} H^{2}(z) w^{j+1} \subset q_{j+1} H^{2}(z) w^{j+1},
$$

and this shows that $\bigoplus_{j=0}^{\infty} q_{i} H^{2}(z) w^{j}$ is also $w$-invariant.

Invariant subspace of the form $\bigoplus_{j=0}^{\infty} q_{i} H^{2}(z) w^{j}$, where $\left\{q_{i}(z): i \geq 0\right\}$ is an inner sequence, shall be said to be inner-sequence-based in this paper, and it is the primary subject of this paper. The above observations indicate that every invariant subspace has a smallest inner-sequence-based invariant subspaces containing it (i.e., an inner-sequence-based envelope). Of course, this envelope is non-trivial only when $q_{0}$ is not a constant, or equivalently, $\overline{\pi_{1}(M)}$ is not dense in $H^{2}(z)$, since if $q_{0}$ is a constant, the inner sequence is a sequence of non-zero scalars and hence

$$
\bigoplus_{j=0}^{\infty} q_{i} H^{2}(z) w^{j}=H^{2}\left(D^{2}\right) \text {. }
$$

Let $M$ be $z$-invariant and $N=H^{2}\left(D^{2}\right) \ominus M$, and we denote the compression of $T_{z}$ to $N$ by $S_{z}$. It is well-known that $S_{z}$ serves as a model for the so-called $C$. 0 class contractions, namely the class of contractions $A$ with $\left(A^{*}\right)^{n}$ converging strongly to 0. Clearly, the $C$. o class is very large, for instance, every strict contraction is in it. If $M$ is invariant (i.e., also invariant for $T_{w}$ ), then $S_{z}$ on $N$ is much less general, but it still represents a good class of interesting operators. For example, the Bergman shift and unilateral shifts with any multiplicity are all unitarily equivalent to $S_{z}$ for $M$ an invariant subspace. For convenience, we let $\mathcal{S}$ denote the collection of operators that are unitarily equivalent to $S_{z}$ or $S_{w}$ on $N=H^{2}\left(D^{2}\right) \ominus M$ for some invariant subspace $M$; here $S_{w}$ is the compression of $T_{w}$ to $N$. In Section 2 , we will show that every Jordan operator is in $\mathcal{S}$. In Section 3, we show that Rudin's invariant subspace (cf. $[\mathrm{Ru}]$ ), formerly believed to be pathological, is in fact innersequence-based. The core operator is an important associate of invariant subspaces of $H^{2}\left(D^{2}\right)$ (cf. [GY]). In Section 4 we show how one can compute the core operator explicitly for the case of an inner-sequence-base invariant subspace. Calculations in Section 4 lead to an interesting upper bound for $\sum_{j} 1-\left|\lambda_{j}\right|$, where $\left\{\lambda_{j}\right\}$ are zeros of a Blaschke product. We will address this point in Section 5 . 


\section{A NOTE ON JORDAN OPERATORS}

Let $H^{2}(D)$ be the Hardy space over the unit disk. Multiplication by coordinate function $z$ on $H^{2}(D)$ is the unilateral shift, and its invariant subspace is of the form $\theta H^{2}(D)$, where $\theta$ is an inner function. The compression $S(\theta)$ of the unilateral shift to the quotient space $N_{\theta}:=H^{2}(D) \ominus \theta H^{2}(D)$ is called a Jordan block. To be precise, $S(\theta) f=P_{N_{\theta}} z f, \quad f \in N_{\theta}$, where $P_{N_{\theta}}$ is the projection from $H^{2}(D)$ onto $N_{\theta}$. For an inner sequence $\left\{q_{j}(z): 0 \leq j \leq m\right\}$, the direct sum $\bigoplus_{j=0}^{m} S\left(q_{j}\right)$ is often called a Jordan operator. A celebrated result in the 70's is that every $C_{0}$ class operator on a separable Hilbert space is quasisimilar to a Jordan operator (cf. $\mathrm{Be}]$.

Jordan operators are directly connected with inner-sequence-based invariant subspaces. For an inner sequence $\left\{q_{j}(z): j \geq 0\right\}$, and $M=\bigoplus_{j=0}^{\infty} q_{j} H^{2}(z) w^{j}$, we observe that:

$$
N=\left(\bigoplus_{j=0}^{\infty} H^{2}(z) w^{j}\right) \ominus\left(\bigoplus_{j=0}^{\infty} q_{j} H^{2}(z) w^{j}\right)=\bigoplus_{j=0}^{\infty}\left(H^{2}(z) \ominus q_{j} H^{2}(z)\right) w^{j} .
$$

Let $P_{j}$ denote the orthogonal projection from $H^{2}\left(D^{2}\right)$ onto $\left(H^{2}(z) \ominus q_{j} H^{2}(z)\right) w^{j}$. Clearly, $P_{N}=\bigoplus_{j=0}^{\infty} P_{j}$. For every $f=\sum_{j=0}^{\infty} f_{j}(z) w^{j} \in N$, where $f_{j} \in H^{2}(z) \ominus$ $q_{j} H^{2}(z)$

$$
\begin{aligned}
S_{z} f & =\left(\bigoplus_{j=0}^{\infty} P_{j}\right)\left(\sum_{i=0}^{\infty} z f_{i}(z) w^{j}\right) \\
& =\sum_{i=0}^{\infty}\left(\bigoplus_{j=0}^{\infty} P_{j} z f_{i}(z) w^{i}\right) \\
& =\sum_{i=0}^{\infty} P_{i} z f_{i}(z) w^{i} \\
& =\sum_{i=0}^{\infty}\left(S\left(q_{i}\right) f_{i}\right) w^{i} .
\end{aligned}
$$

So with respect to the decomposition $N=\bigoplus_{j=0}^{\infty}\left(H^{2}(z) \ominus q_{j} H^{2}(z)\right) w^{j}$,

$$
S_{z}=\bigoplus_{j=0}^{\infty} S\left(q_{j}\right)
$$

and we obtain the following fact.

Corollary 2.1. Every Jordan operator is in $\mathcal{S}$.

It is not hard to see that if $q_{j}$ is a scalar starting from $j=k$, then the direct sum above is a finite sum of $k$ terms.

It is also worth noting that (2-1) gives a necessary condition for an invariant subspace to be of inner-sequence-based type. For instance, (2-1) implies that $\sigma\left(S_{z}\right) \cap$ $D$, being the zeros of $S\left(q_{0}\right)$, is discrete. One can easily come up with invariant subspaces for which $\sigma\left(S_{z}\right) \cap D$ is not discrete. For example, if we let $M=[z-w]$ be the closure of the ideal $(z-w)$ in the polynomial ring $C[z, w]$, then $S_{z}$ on $H^{2}\left(D^{2}\right) \ominus M$ is equivalent to the Bergman shift, and hence $\sigma\left(S_{z}\right)=\bar{D}$. This indicates that $[z-w]$ is not inner sequence based. It is not difficult to check that the envelope of $[z-w]$ is $z H^{2}(z) \oplus \bigoplus_{j=1}^{\infty} H^{2}(z) w^{j}$. 


\section{RUDIN'S INVARIANT SUBSPACE}

In $[\mathrm{Ru}$, Rudin constructed an invariant subspace of infinite rank as follows. Let $M$ be the Hardy invariant subspace consisting of all functions in $H^{2}\left(D^{2}\right)$ which have a zero of order greater than or equal to $n$ at $\left(\alpha_{n}, 0\right)=\left(1-n^{-3}, 0\right)$ for any positive integer $n$. For quite a long time this invariant subspace was viewed as somewhat pathological. Here, we will show that it is in fact an inner-sequence-based invariant subspace. It is interesting because this means that Rudin's invariant subspace, despite the fact that it has infinite rank, has a very simple structure.

For simplicity, we denote $H^{2}\left(D^{2}\right)$ by $H^{2}$ and set $b_{n}(z)=\left(z-\alpha_{n}\right) /\left(1-\alpha_{n} z\right)$. Then we have $M=\bigcap_{n \geq 1} M_{n}$, where

$$
M_{n}=b_{n}^{n}(z) H^{2} \vee b_{n}^{n-1}(z) w H^{2} \vee \cdots \vee w^{n} H^{2},
$$

that is, $M_{n}$ is the invariant subspace consisting of all functions in $H^{2}$ which have a zero of order greater than or equal to $n$ at $\left(\alpha_{n}, 0\right)$. Further, we have

$$
\begin{aligned}
M_{n} & =b_{n}^{n}(z) H^{2}(z) \oplus b_{n}^{n-1}(z) w H^{2}(z) \oplus \cdots \oplus w^{n} H^{2} \\
& =\sum_{k=0}^{n-1} \oplus b_{n}^{n-k}(z) w^{k} H^{2}(z) \oplus w^{n} H^{2} .
\end{aligned}
$$

We define a family of inner functions inductively as follows:

$$
\left\{\begin{array}{l}
q_{0}(z)=\prod_{n=1}^{\infty} b_{n}^{n}(z), \\
q_{j}(z)=q_{j-1}(z) / \prod_{n=j}^{\infty} b_{n}(z) \quad(j \geq 1) .
\end{array}\right.
$$

Then $q_{j}(z)$ is divisible by $q_{j+1}(z)$ for every $j \geq 0$, and we have

$$
M=\bigcap_{n \geq 1} M_{n}=\bigoplus_{j=0}^{\infty} q_{j}(z) H^{2}(z) w^{j} .
$$

The fact that Rudin's example has infinite rank prompts the following conjecture.

Conjecture. If $\left\{q_{j}(z)\right\}$ is an inner sequence such that $q_{j+1}$ is a proper factor of $q_{j}$ for each $j \geq 0$, then $\bigoplus_{j=0}^{\infty} q_{j} H^{2}(z) w^{j}$ has infinite rank.

\section{THE CORE OPERATOR}

For a invariant subspace $M$, we let $\left(R_{z}, R_{w}\right)$ denote the restriction of $\left(T_{z}, T_{w}\right)$ to $M$. So it is clear that $\left(R_{z}, R_{w}\right)$ is a pair of commuting isometries. The core operator $C$ for $M$ is defined as

$$
C=I-R_{z} R_{z}^{*}-R_{w} R_{w}^{*}+R_{z} R_{w} R_{z}^{*} R_{w}^{*} .
$$

A parallel associate for $\left(S_{z}, S_{w}\right)$ is

$$
\Delta_{S}:=I-S_{z}^{*} S_{z}-S_{w}^{*} S_{w}+S_{z}^{*} S_{w}^{*} S_{z} S_{w}
$$

Both $C$ and $\Delta_{S}$ are useful tools in the study of invariant subspaces. We refer the readers to $\mathrm{GY}$ ] and [Ya2 for details. In this section, we will see that both $C$ and $\Delta_{S}$ can be written as a direct sum with respect to the decomposition $M=$ $\bigoplus_{j=0}^{\infty} q_{j}(z) H^{2}(z) w^{j}$. For simplicity we let $q_{j}^{\prime}=\frac{q_{j-1}}{q_{j}}, j=1,2,3, \ldots$ 
Let $g=q_{j} f_{j} w^{j}$ be any function in $q_{j} H^{2}(z) w^{j}$, where $j \geq 1$. Then

$$
\begin{aligned}
R_{w}^{*}\left(q_{j} f_{j} w^{j}\right) & =\left[\left(I-P_{j-1}\right)\left(q_{j} f_{j}\right)\right] w^{j-1} \\
& =\left(\sum_{i=0}^{\infty}\left\langle q_{j} f_{j}, q_{j-1} z^{i}\right\rangle q_{j-1} z^{i}\right) w^{j-1}
\end{aligned}
$$

and

$$
\begin{aligned}
\left(I-R_{z} R_{z}^{*}\right) g & =\sum_{i=0}^{\infty}\left\langle g, q_{i} w^{i}\right\rangle q_{i} w^{i} \\
& =\left\langle q_{j} f_{j} w^{j}, q_{j} w^{j}\right\rangle q_{j} w^{j} \\
& =f_{j}(0) q_{j} w^{j} .
\end{aligned}
$$

It follows that

$$
\begin{aligned}
C g & =\left[I-R_{z} R_{z}^{*}-R_{w}\left(I-R_{z} R_{z}^{*}\right) R_{w}^{*}\right] g \\
& =f_{j}(0) q_{j} w^{j}-\left\langle q_{j} f_{j}, q_{j-1}\right\rangle q_{j-1} w^{j} \\
& =\left(\left\langle f_{j}, 1\right\rangle 1-\left\langle f_{j}, q_{j}^{\prime}\right\rangle q_{j}^{\prime}\right) q_{j} w^{j} .
\end{aligned}
$$

This shows that $q_{j} H^{2}(z) w^{j}$ is invariant for $C$ and on $q_{j} H^{2}(z) w^{j}$

$$
C \cong 1 \otimes 1-q_{j}^{\prime} \otimes q_{j}^{\prime} .
$$

Moreover, since $R_{w}^{*}$ is 0 on $q_{0} H^{2}(z)$, it is easy to verify that $C \cong 1 \otimes 1$ on $q_{0} H^{2}(z)$. Summarizing these observations we have

Corollary 4.1. With respect to the decomposition $M=\bigoplus_{j=0}^{\infty} q_{j} H^{2}(z) w^{j}$,

$$
C \cong 1 \otimes 1 \bigoplus_{j=1}^{\infty}\left(1 \otimes 1-q_{j}^{\prime} \otimes q_{j}^{\prime}\right) .
$$

Now we take a look at $\Delta_{S}$. Let $f_{j}$ be any function in $H^{2}(z) \ominus q_{j} H^{2}(z)$, where $j \geq$ 1. Then as indicated before, $S_{z}\left(f_{j} w^{j}\right)=\left(S\left(q_{j}\right) f_{j}\right) w^{j}$, and $S_{w}\left(f_{j} w^{j}\right)=\left(P_{j+1} f_{j}\right) w^{j}$. Writing

$$
\Delta_{S}=\left(I-S_{z}^{*} S_{z}\right)-S_{w}^{*}\left(I-S_{z}^{*} S_{z}\right) S_{w}
$$

and $I_{j}-S^{*}\left(q_{j}\right) S\left(q_{j}\right)$ as $D_{j}$, where $I_{j}$ stands for the identity map on $H^{2}(z) \ominus q_{j} H^{2}(z)$, we compute that

$$
\begin{aligned}
\Delta_{S}\left(f_{j} w^{j}\right) & =w^{j}\left(I_{j}-S^{*}\left(q_{j}\right) S\left(q_{j}\right)\right) f_{j}-S_{w}^{*}\left(w^{j+1}\left(I_{j+1}-S^{*}\left(q_{j+1}\right) S\left(q_{j+1}\right)\right)\left(P_{j+1} f_{j}\right)\right) \\
& =w^{j} D_{j} f_{j}-w^{j} D_{j+1} P_{j+1} f_{j} \\
& =w^{j} D_{j} f_{j}-w^{j} D_{j+1}\left(P_{j}-\left(P_{j}-P_{j+1}\right)\right) f_{j} .
\end{aligned}
$$

One verifies that $\left.D_{j+1}\left(P_{j}-P_{j+1}\right)\right)=0$, and hence

$$
\Delta_{S}\left(f_{j} w^{j}\right)=\left(D_{j}-D_{j+1}\right) f_{j} w^{j}, \quad j \geq 0 .
$$

We summarize these observations in the following corollary.

Corollary 4.2. With respect to the decomposition $N=\bigoplus_{j=0}^{\infty}\left(H^{2}(z) \ominus q_{j} H^{2}(z)\right) w^{j}$,

$$
\Delta_{S} \cong \bigoplus_{j=0}^{\infty}\left(D_{j}-D_{j+1}\right) .
$$


It is well-known that $D_{j}$ is of rank 1 when $q_{j}$ is non-trivial, and it can be explicitly expressed in terms of $q_{j}$. We will return to this point in Section 5.

Lemma 4.3. Let $f$ and $g$ be non-zero functions in $H^{2}(z)$ and $A=f \otimes f-g \otimes g$. Then

$$
\operatorname{tr} A^{2}=\|f\|^{4}+\|g\|^{4}-2|\langle f, g\rangle|^{2} .
$$

Proof. It is a simple calculation. Clearly, $A$ is selfadjoint. Since

$$
A f=\|f\|^{2} f-\langle f, g\rangle g, \quad A g=\langle g, f\rangle f-\|g\|^{2} g,
$$

with respect to the basis $\{f, g\}, A$ has the matrix form

$$
\left(\begin{array}{ll}
\|f\|^{2} & \langle g, f\rangle \\
-\langle f, g\rangle & -\|g\|^{2}
\end{array}\right)
$$

If we denote the eigenvalues of $A$ by $\lambda_{1}$ and $\lambda_{2}$, then

$$
\lambda_{1}+\lambda_{2}=\|f\|^{2}-\|g\|^{2}, \quad \lambda_{1} \lambda_{2}=-\|f\|^{2}\|g\|^{2}+|\langle f, g\rangle|^{2} .
$$

So

$$
\operatorname{tr} A^{2}=\lambda_{1}^{2}+\lambda_{2}^{2}=\|f\|^{4}+\|g\|^{4}-2|\langle f, g\rangle|^{2} .
$$

The following fact follows directly from Corollary 4.1 and the above lemma.

Corollary 4.4. $\operatorname{tr} C^{2}=1+2 \sum_{j=1}^{\infty} 1-\left|q_{j}^{\prime}(0)\right|^{2}$.

Recall that for an invariant subspace $M$, its fringe operator $F$ is defined on $M \ominus z M$ by

$$
F f=P_{M \ominus z M} w f, \quad f \in M \ominus z M .
$$

It is indicated in Ya1 that the fringe operator is also a very useful tool for the study in this area. It is interesting to see how the fringe operator acts on $M=$ $\bigoplus_{j=0}^{\infty} q_{j} H^{2}(z) w^{j}$. It is not difficult to check that in this case

$$
M \ominus z M=\bigoplus_{j=0}^{\infty} \mathbb{C} q_{j}(z) w^{j},
$$

and clearly $\left\{q_{j}(z) w^{j}: j \geq 0\right\}$ is an orthonormal basis for $M \ominus z M$. For every $j$,

$$
\begin{aligned}
F\left(q_{j} w^{j}\right) & =P_{M \ominus z M} q_{j} w^{j+1} \\
& =\left\langle q_{j} w^{j+1}, q_{j+1} w^{j+1}\right\rangle q_{j+1}(z) w^{j+1} \\
& =\left\langle q_{j+1} q_{j+1}^{\prime}, q_{j+1}\right\rangle q_{j+1}(z) w^{j+1} \\
& =q_{j+1}^{\prime}(0) q_{j+1}(z) w^{j+1} .
\end{aligned}
$$

This shows that the fringe operator in this case is a weighted shift with weighs

$$
q_{1}^{\prime}(0), q_{2}^{\prime}(0), q_{3}^{\prime}(0), \cdots
$$




\section{An inequality about Blaschke products}

For any bounded linear operator $A$ on a Hilbert space $H$, the so-called minimum modulus

$$
\gamma(A):=\inf \left\{\|A x\|: x \in(\operatorname{ker} A)^{\perp},\|x\|=1\right\}
$$

measures the norm of $A$ 's "partial inverse". Clearly, $A$ has closed range if and only if $\gamma(A)>0$. When $A$ is invertible, $\gamma^{-1}(A)=\left\|A^{-1}\right\|$.

It is shown in Ya2 that for every invariant subspace $M$

$$
\operatorname{tr} C^{2} \leq 2 \gamma^{-2}\left(S_{z}\right)+2 \operatorname{dim} \operatorname{ker}\left(S_{z}\right)-1 .
$$

It is interesting to see what this inequality means for an inner-sequence-based invariant subspace. Without loss of generality we assume $q_{0}(0) \neq 0$. Then by $(2-1)$, $\operatorname{ker}\left(S_{z}\right)$ is trivial. To calculate $\gamma\left(S_{z}\right)$, we consider a general Jordan operator $S(\theta)$ on the space $N_{\theta}:=H^{2}(D) \ominus \theta H^{2}(D)$. One verifies that

$$
I-S^{*}(\theta) S(\theta)=P_{N_{\theta}}(\bar{z} \theta) \otimes P_{N_{\theta}}(\bar{z} \theta) .
$$

Therefore, for $g \in N_{\theta}$,

$$
\begin{aligned}
\|S(\theta) g\|^{2} & =\|g\|^{2}-\left|\left\langle g, P_{N_{\theta}}(\bar{z} \theta)\right\rangle\right|^{2} \\
& \geq\left(1-\left\|P_{N_{\theta}}(\bar{z} \theta)\right\|^{2}\right)\|g\|^{2} \\
& =|\theta(0)|^{2}\|g\|^{2},
\end{aligned}
$$

and the equality is obtained when $g=P_{N_{\theta}}(\bar{z} \theta)$. This shows that $\gamma(S(\theta))=|\theta(0)|$. (2-1) then implies that

$$
\gamma\left(S_{z}\right)=\inf \left\{\left|q_{j}(0)\right|: j \geq 0\right\} .
$$

But since every $q_{j}$ is a factor of $q_{0}$,

$$
\gamma\left(S_{z}\right)=\left|q_{0}(0)\right|
$$

Combining Corollary 4.4, (5-1) and (5-2), we have

$$
\sum_{j=1}^{\infty} 1-\left|q_{j}^{\prime}(0)\right|^{2} \leq\left|q_{0}(0)\right|^{-2}-1 .
$$

Or equivalently, we have

Corollary 5.1. For $M=\bigoplus_{j=0}^{\infty} q_{j} H^{2}(z) w^{j}, \quad \operatorname{tr} C^{2} \leq 2\left|q_{0}(0)\right|^{-2}-1$.

If we let $B(z)$ be a Blaschke product with zeros $\left\{\lambda_{j}: 1 \leq j \leq m\right\}$ counting multiplicity, where $m$ can be infinity, and set

$$
q_{j}=\prod_{i=j+1}^{m} \frac{\lambda_{i}-z}{1-\overline{\lambda_{i}} z}, \quad j \geq 0,
$$

then (5-3) leads to an interesting inequality for Blaschke products, namely

$$
\sum_{j=1}^{\infty} 1-\left|\lambda_{j}\right|^{2} \leq|B(0)|^{-2}-1 .
$$

Let us pursue this point a little further. Clearly, (5-4) is equivalent to the general inequality

$$
\sum_{j=1}^{\infty} 1-\left|\lambda_{j}\right|^{2} \leq\left(\prod_{j=1}^{\infty}\left|\lambda_{j}\right|\right)^{-2}-1
$$


for any sequence $\left\{\lambda_{j}: j \geq 1\right\} \subset \bar{D}$ which can be proved easily by induction, and the equality holds only in the case when both sides are zero. So substituting $\lambda_{j}$ by its square root, (5-4) leads to the following inequality.

$$
\sum_{j} 1-\left|\lambda_{j}\right| \leq|B(0)|^{-1}-1
$$

where $B(z)$ is a Blaschke product with zeros $\left\{\lambda_{j}\right\}$ counting multiplicity. (5-5) is a simple inequality, but surprisingly it appears to have been unknown before. If $f \in H^{p}(D)$, where $1 \leq p \leq \infty$, and $\|f\|_{p} \leq 1$, and $\left\{\lambda_{j}: j \geq 1\right\}$ are its zeros counting multiplicity, then $B$ is a factor of $f$, and $|B(0)| \geq|f(0)|$. So (5-5) can be generalized to the following corollary.

Corollary 5.2. If $f \in H^{p}(D)$ with $\|f\|_{p} \leq 1$, where $1 \leq p \leq \infty$, and $\left\{\lambda_{j}: j \geq 1\right\}$ are its zeros counting multiplicity, then

$$
\sum_{j=1}^{\infty} 1-\left|\lambda_{j}\right| \leq|f(0)|^{-1}-1 .
$$

If $\|f\|_{\infty} \leq 1$, and $\eta \in D$, then $\frac{f(z)-\eta}{1-\bar{\eta} f(z)}$ is also a analytic self map of $D$, and hence Corollary 5.2 implies

$$
\sum_{f(z)=\eta} 1-|\eta| \leq\left|\frac{1-\bar{\eta} f(0)}{f(0)-\eta}\right|-1 .
$$

\section{ACKNOWLEDGMENTS}

The authors wish to express their gratitude to K. Izuchi whose discussions with the authors have nurtured an important part of Section 3 and Section 5. Thanks go to R. O'Neil for his valuable input. The authors also thank H. Bercovici and D. Sarason for references on the inequality (5-5).

\section{REFERENCES}

[Be] H. Bercovici, Operator theory and arithmetic in $H^{\infty}$, Mathematical Surveys and Monographs, No. 26, A.M.S. 1988, Providence, Rhode Island. MR0954383 (90e:47001)

[DM] R. G. Douglas and G. Misra, On quotient modules, Recent advances in operator theory and related topics (Szeged, 1999), 203-209, Oper. Theory Adv. Appl., 127, Birkhäuser, Basel, 2001. MR.1902802 (2003c:46022)

[DP] R. G. Douglas and V. Paulsen, Hilbert modules over function algebras, Pitman Research Notes in Mathematics Series 217, Longman Scientific and Technical, 1989. MR1028546 (91g:46084)

[GY] K. Guo and R.Yang, The core function of submodules over the bidisk, Indiana Univ. Math. J. 53 (2004), 205-222. MR2048190 (2005m:46048)

[Ru] W. Rudin, Function theory in polydiscs, Benjamin, New York, 1969. MR0255841 (41:501)

[Ya1] R. Yang, Operator theory in the Hardy space over the bidisk (III), J. of Funct. Anal. 186, 521-545 (2001). MR:1864831(2002m:47008)

[Ya2] R. Yang, On two variable Jordan blocks (II), Inte. Equ. Oper. Theory. 56 (2006), 431-449.

Department of Mathematics, Kanagawa University, Yokohama, Japan

E-mail address: seto@kanagawa-u.ac.jp

Department of Mathematics and Statistics, SUny at Albany, Albany, New York 12222

E-mail address: ryang@math.albany.edu 